Cytori, GSK, Genentech/Roche, Galapagos, Employee of: Elcos Sciences, Inc, Cathie Spino: None declared, Vivek Nagaraja: None declared, Robin Domsic Consultant for: Eicos Sciences Inc/Civi Biopharma and Boehringer-Ingelheim., Robert Lafyatis: None declared, Viginia Steen Consultant for: CSL Behring, Corbus and Bayer, Paid instructor for: Advisory Board: CSL Behring, Roach, Bayer and Reata, Jessica Gordon Grant/research support from: Corbus Pharmaceuticals

Cumberland Pharmaceuticals, Tracy Frech: None declared, Pei-Suen Tsou: None declared

DOI: 10.1136/annrheumdis-2019-eular.2122

\section{SAT0280 ESOPHAGEAL DYSMOTILITY AND PULMONARY DISEASE IN PATIENTS WITH SCLERODERMA: A RETROSPECTIVE STUDY}

Navya Kuchipudi ${ }^{1}$, Shweta Kishore ${ }^{2}$, Chia-Ling Kuo ${ }^{3}$,

Santhanan Lakshminarayanan ${ }^{1}$,Ranadeep Mandhadi'. ${ }^{1}$ University of Connecticut Rheumatology, Farmington, United States of America; ${ }^{2}$ University of Mississippi Medical Center, Jackson, United States of America; ${ }^{3}$ University of Connecticut, Community Medicine and Health Care, Farmington, United States of America

Background: Systemic sclerosis (SSc) is a connective tissue disease with pulmonary involvement seen in $75 \%$ of patients and esophageal involvement in $90 \%$ of the patients. Pulmonary disease has overtaken renal disease as the leading cause of death (1). There is conflicting evidence about the association between esophageal dysmotility and lung involvement (Interstitial Lung Disease (ILD) and Pulmonary Artery Hypertension $(\mathrm{PAH}))$.

Objectives: Our aim was to evaluate the relationship between esophageal dysmotility and lung disease by correlating the results of Echocardiogram and Pulmonary Function Test (PFT) with Esophageal Transit Study (ETT).

Methods: Charts of SSc patients fulfilling 2013 ACR/EULAR classification criteria seen in Rheumatology clinic from 2004 to 2015 were reviewed. Patient demographics, ETT result, FVC and DLCO data from PFT as well as pulmonary pressures from echocardiogram were collected at baseline, years $1,3,5$ and 10. Patients were divided based on their initial ETT findings into normal and abnormal ETT groups. Using logistic regression models, we compared elevated PASP with ETT status at baseline, and years 3 and 5 . Linear mixed effects model was used to adjust for covariates (disease subtype, smoking, obesity, use of immunomodulator) when analyzing PFT outcome with time period and ETT status

Results: 130 patients were identified with either Limited Cutaneous SSc (109) or Diffuse Cutaneous SSc (21) with a mean age of 52.65 years \pm 12.59. ETT was normal in $67(52 \%)$ and abnormal in $63(48 \%)$ patients. Number of patients with abnormal PASP was not statistically different between the two groups ( $p$ values $0.104,0.178,0.653$ at baseline, years 3 and 5 respectively). Likewise, the odds ratio for abnormal PASP was 2.75 in patients with abnormal ETT compared to normal ETT, but the results were not statistically significant ( $p: 0.428$ ), and the odds ratio did not vary with time ( $p$ : 0.731 ). Sample size was too small to conduct separate longitudinal analysis for adjusted models. The mean DLCo was statistically worse in abnormal ETT patients $[p$ value $<0.0001$ and 0.0004 for unadjusted and adjusted models] as were the progression rates per year for DLCo at -1.95 ( $p$-value: 0.023 ) and -2.25 ( $p$-value: 0.019) for unadjusted and adjusted models respectively. The Mean FVC was not statistically different between the two groups, although it's progression rate per year was statistically worse only in the adjusted model ( $p$ value: 0.018).

Conclusion: Esophageal dysmotility was associated with increased pulmonary involvement in the form of abnormal DLCo with worsening progression rates per year. Interestingly, neither PASP nor FVC were statistically different even though progression rate for FVC was worse in adjusted models. We postulate that the small sample size for Echo and differences in the factors determining DLCo vs. FVC and PASP explains the disparity in the results. Larger prospective trials are needed to investigate if there is a causal relationship between esophageal dysfunction and pulmonary involvement.

\section{REFERENCE}

[1] Mathai, S. C., Hummers, L. K. (2009), Survival in pulmonary hypertension associated with the scleroderma spectrum of diseases: Impact of interstitial lung disease. Arthritis \& Rheumatism, 60: 569-577. doi:10.1002/art.24267
Disclosure of Interests: None declared

DOI: 10.1136/annrheumdis-2019-eular.1843

\section{SAT0281 RELATIONSHIP BETWEEN AUTOANTIBODIES AND CYTOKINE PROFILES IN PATIENTS WITH MYOSITIS}

Kazuhihiro Kurasawa, Satoko Arai, Yuta Takamura, Tomoyuki Miyao,

Ayae Tanaka, Ryutaro Yamazaki, Takayoshi Owada, Reika Maezawa

Masafumi Arima. Dokkyo Medical University, Rheumatology, Mibu, Tochigi, Japan

Background: By autoantibodies, myositis is recently classified into subgroups with distinctive clinical features. Cytokines play critical roles in the development of the clinical features. However, it is not fully unknown whether myositis subgroups defined by autoantibodies have unique cytokine profiles.

Objectives: To clarify the relationship between cytokine profiles and autoantibodies in myositis.

Methods: Subjects were myositis patients who admitted Dokkyo Medical University and whose serum before starting therapy were available. Serum cytokine levels (IL-1a, 1b, 2, 4,5, 6, 10,12, 13,15,17, 23,IFN-g TNF-a, IFN-a, b,IP-10 and MCP-1) in sera from the patients and controls were measured using Q-Plex ${ }^{\mathrm{TM}}$ Multiplex Arrays. Demographic and clinical data were obtained by reviewing medical record retrospectively.

Results: Subjects were 45 myositis patients with male/female; 22/23 and 59.4 years of mean age. Amyopathic dermatomyositis (ADM), classica $\mathrm{DM}$ and polymyositis (PM) were 21,11 and12 cases, respectively. Autoantibodies were anti-MDA5+ in 21, anti-ARS + in18 and anti-TIF1g+ in 5 cases. ILD and malignancy was complicated in 39 and 5 cases, respectively.

Serum cytokine levels were measured. As shown in Fig.1, IP-10 levels were increased in all types of myositis patients, but not in controls. In ant-MDA5 Ab + myositis, IL-6, IL-10, IL-15, TNF-a, IFN-a and MCP-1 levels were increased. In anti-ARS $A b+$ cases, IFN-b levels were elevated as well as TNF-a and MCP-1.

Through the correlation analysis between cytokine levels, cytokine groups were identified (Fig.2). In anti-MDA5 $\mathrm{Ab}+$ cases, 2 cytokine groups were identified; one includes IFN-a, IL-15, TNF-a and IL-10, and the other involved IL-6, MCP-1. Both groups connected with IP-10. In In anti-ARS

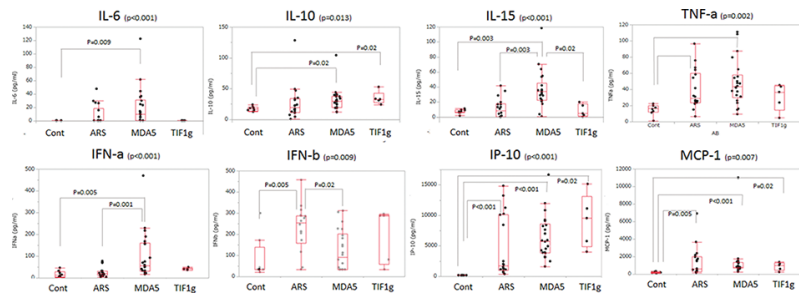

Figure 1. Serum cytokine levels in myositis

ARS

MDA5

IFNb
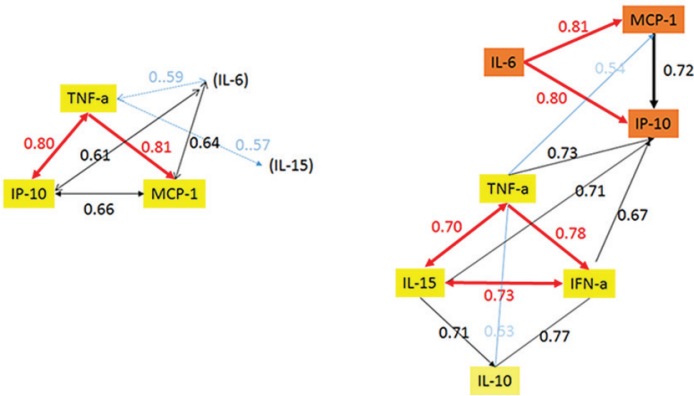

Figure 2. Cytokine networks in a-ARS ab+ and a-MDA5 ab+ myositis 
$\mathrm{Ab}+$ cases, 2 groups were found; one included TNF-a, MCP-1and IP-10, and the other had IFN-b as a sole component.

Conclusion: Cytokine profiles differ among subgroups in myositis. In antiDDA5+ myositis, IL-15 and IFN-a with strong correlation and IL-6 were characteristic cytokines, while IFN-b was a unique one in anti-ARS+ myositis

Disclosure of Interests: None declared

DOI: 10.1136/annrheumdis-2019-eular.5542

\section{SAT0282 EFFICACY AND SAFETY OF ORAL PROSTACYCLIN RECEPTOR AGONIST SELEXIPAG IN PATIENTS WITH SYSTEMIC SCLEROSIS -ASSOCIATED PULMONARY ARTERIAL HYPERTENSIONIN DAILY CLINICAL PRACTICE, A CASE SERIES}

Jacqueline Lemmers $^{1}{ }^{1}$, Håvard Fretheim ${ }^{2}$, Hanneke Knaapen-Hans ${ }^{1}$, Frank van den Hoogen ${ }^{1}$, Jolanda van Haren-Willems ${ }^{1}$, Toon Duijnhouwer ${ }^{1}$, C.H.M. van den Ende ${ }^{1}$, Arie van Dijk ${ }^{1}$,Anna-Maria Hoffmann-Vold ${ }^{2}$, Madelon Vonk ${ }^{1} .{ }^{1}$ Radboud University Medical Centre, Nijmegen, Netherlands; ${ }^{2}$ Oslo University Hospital, Oslo, Norway

Background: Pulmonary arterial hypertension $(\mathrm{PAH})$ is a frequent complication in systemic sclerosis (SSc) and a major cause of death despite the available treatments (1). Until the availability of the oral prostacyclin receptor agonist selexipag in 2016, parenteral medication targeting the prostacyclin pathway was often not feasible for patients with SSc -PAH due to practical barriers. Selexipag added to background therapy improves prognosis of $\mathrm{PAH}$ in the published pivotal trial (2). However, to date information on results of SSc-PAH patients treated with any combination of targeted therapies including selexipag in daily clinical practice are lacking.

Objectives: Evaluation of the efficacy and safety of selexipag in SSc$\mathrm{PAH}$ patients in a real life case series.

Methods: All patients with SSc associated $\mathrm{PAH}$ (WHO group 1) diagnosed by right heart catheterization who started with selexipag between 09-2016 and 06-2018 from two PAH expert centres were included. Data on demographics, concurrent medication, functional class, 6 minute walking distance and NT-pro-BNP level were recorded at baseline and during follow-up. Every 12 weeks we recorded the opinion of the treating expert team, resulting in a conclusion "improvement", "stabilization" or "deterioration". Additionally treatment effect was assessed according to the abbreviated risk assessment described by Boucly (3). Side effects and adverse events were registered and evaluated.

Results: We included 13 SSc-PAH patients, all classified as limited cutaneous SSc. Ten patients were female. Median age was 68 years (IQR 58-75), median SSc disease duration 7.4 years (IQR 4.7-13.5) and median PAH duration 4 years (IQR 2.5-7.5). All patients were on double background therapy. At baseline 1 patient had two low risk criteria, 1 patient had one low risk criterion, the other patients had no low risk criteria. Two patients discontinued selexipag within 4 weeks due to side effects. The eleven patients reaching maintenance dosage had a median follow up of 48 weeks (IQR 24-72). At the end of follow up, 2/11 patients improved, 4/11 stabilized, and 5/11 deteriorated as evaluated by their expert team. Two of these deteriorated patients died due to right ventricular failure and gastro-intestinal bleeding respectively. Of the patients deteriorating, 3 initially stabilized for a period of 24 to 60 weeks. None of the patients achieved 3 low risk criteria, three patients achieved 1 low risk criterion. No unknown side effects were reported.

Conclusion: Adding selexipag to double background therapy in a high risk cohort stabilized symptoms in the majority of these patients with an acceptable safety profile. Sustained improvement of $\mathrm{PAH}$ symptoms is reached in only a minority of our SSc-PAH patients. Further research examining multiple target therapy, including selexipag, in patients with early SSc-associated PAH is warranted.

\section{REFERENCES}

[1] Lefevre G, et al. Survival and prognostic factors in systemic sclerosisassociated pulmonary hypertension: a systematic review and meta-analysis. Arthritis Rheum. 2013;65(9):2412-23.

[2] Sitbon O, et al. Selexipag for the Treatment of Pulmonary Arterial Hypertension. N Engl J Med. 2015;373(26):2522-33.

[3] Boucly A, et al. Risk assessment, prognosis and guideline implementation in pulmonary arterial hypertension. Eur Respir J. 2017;50(2).
Disclosure of Interests: Jacqueline Lemmers: None declared, Håvard Fretheim Grant/research support from: Travel bursary from Actelion and GSK, Hanneke Knaapen-Hans: None declared, Frank van den Hoogen: None declared, Jolanda van Haren-Willems: None declared, Toon Duijnhouwer: None declared, C.H.M. van den Ende: None declared, Arie van Dijk Grant/research support from: Unrestricted grant for PhD student from Actelion, Consultant for: Actelion, Speakers bureau: Actelion, Anna-Maria Hoffmann-Vold Grant/research support from: Received research funding or other remuneration from Boehringer Ingelheim, GSK, and Actelion, Consultant for: Received consulting fees or other remuneration from Boehringer Ingelheim, GSK, and Actelion, Speakers bureau: Actelion and Boehringer Ingelheim, Madelon Vonk Grant/research support from: Madelon Vonk has received unrestricted research funds from Actelion and Therabel, Consultant for: Madelon Vonk was a consultant for Actelion, Boehringer-Ingelheim, Speakers bureau: Actelion, Boehringer-Ingelheim, Roche DOI: 10.1136/annrheumdis-2019-eular.3021

\section{SAT0283 CLINICAL AND SEROLOGICAL FEATURES OF SYSTEMIC SCLEROSIS IN ITALIAN AND EGYPTIAN PATIENTS}

Vasiliki Liakouli ${ }^{1}$, Hesham Hamoud ${ }^{2}$, Piero Ruscitti ${ }^{1}$, Mahmoud Ibrahim Risha ${ }^{3}$, Mohamed Elsayed Hanafy ${ }^{3}$, Khaled Elnoafly ${ }^{3}$, Rasha Ali ${ }^{4}$, Gihan Omar ${ }^{4}$, Adel Elsayed $^{5}$, Abdelazeim Elhefny ${ }^{5}$, Mervat Abogabal ${ }^{5}$, Sameh Mobasher ${ }^{5}$, Paola Cipriani ${ }^{1}$, Roberto Giacomelli'. ' University of L'Aquila, L'Aquila, Italy, ${ }^{2}$ University of Al Azhar, Cairo, Egypt, ${ }^{3}$ Al Azhar University, Cairo, Egypt, ${ }^{4}$ Minia University, Cairo, Egypt, ${ }^{5}$ Ain Shams University, Cairo, Egypt

Background: Clinical and serological variations exist in the severity of Systemic Sclerosis (SSc) patients, according to different geographic areas. Objectives: To evaluate clinical and serological features in 2 cohorts of Italian and Egyptian SSc patients and to identify factors associated with Interstitial lung disease (ILD), a leading cause of mortality, by using multivariate logistic regression analysis.

Methods: An Italian center and 3 Egyptian centers participated in SSc patient recruitment in 2017. The cross-sectional demographic, clinical, and laboratory data were collected and defined according to severity score and activity index, previously developed [1, 2]. The database included 152 consecutive Italian patients, 135 women (88.8\%) and 17 men (11.2\%) and 197 consecutive Egyptian SSc patients, 177 women (89.8\%) and 20 men $(10.2 \%)$, all of whom fulfilled the classification criteria proposed by LeRoy and Medsger [3].

Results: We found that Egyptians SSc patients were younger $(41.18 \pm 12.5$ vs $58.59 \pm 12.6 \mathrm{yrs})$, had an earlier onset of the first non Raynaud's Phenomenon symptom $(7.28 \pm 5.89$ vs $14.99 \pm 11.11)$ and a more severe Modified Rodnan Skin Score (MRSS $>14) \quad(79.27 \%$ vs $13.6 \%)$. A greater percentage of Egyptian patients presented the active form of the disease (53.3\% vs $15.5 \%$ ) and had a Pulmonary Arterial Pressure (PAPs) estimated by echocardiography $\geq 40 \mathrm{mmHg}(37.5 \%$ vs $10.6 \%)$, than Italian patients. Furthermore, Egyptian patients affected from the limited form of the disease (IcSSc), presented a higher MRSS $(67.2 \%$ vs $2.6 \%)$, higher PAPs levels $(34.5 \%$ vs $9.2 \%)$, an active form $(41.7 \%$ vs $7.8 \%)$ and history of past/current ulcers $(62.2 \%$ vs $30.6 \%)$ than Italian IcSSc patients Severity score was higher in Egyptian patients than in the Italians (General: $22.9 \%$ vs $6.2 \%$,Peripheral vascular: $48.2 \%$ vs $23.9 \%$, skin: $19.8 \%$ vs $3.6 \%$, joint/tendon: $19.9 \%$ vs $8.5 \%$, muscle: $8.1 \%$ vs $2.6 \%$, lung: $41.1 \%$ vs $25.3 \%$, heart: $11.2 \%$ vs $4.1 \%$ ). Kidney involvement was more severe in the diffuse cutaneous form (dcSSc) of the disease in Egyptians patients than Italians ( $2 \%$ vs $0.4 \%)$. In the multivariate analysis, PAPs $\geq 40 \mathrm{mmHg}$ (OR: 2.622, 95\%Cl:1.031-6.660, $\mathrm{p}=0.043$ ), past/current ulcers (OR:3.450, 95\%Cl:1.265-9.409, $\mathrm{p}=0.016)$, presence of anticentromere (ACA) (OR:3.846, 95\%Cl:1.488-9.940, $\mathrm{p}=0.005)$ and anti-topoisomerasi (SCL70) (OR:5.012, 95\%Cl:1.740-14.441, $\mathrm{p}=0.003$ ) antibodies and heart involvement (OR:3.018, 95\% $\mathrm{Cl}: 1.448-6.288, \mathrm{p}=0.003$ ) were associated with ILD in the Egyptian database, suggesting a potential role as predictive biomarkers. Advanced age (OR:1.036, 95\%Cl:1.009-1.063, $\mathrm{p}=0.008$ ), dcSSc (OR:4.726, 95\%Cl:2.021-11.049, $\mathrm{p}=0.001$ ) and joint involvement (OR:1.695, 95\%Cl:1.094-2.627, $\mathrm{p}=0.018$ ) were independently associated with ILD in the Italian patients.

Conclusion: This cross-sectional study analyzed the demographic, clinical and laboratoristic variations in Egyptians and Italian patients and their association with ILD, suggesting possible differences to be further investigated in adequately powered studies. 